\title{
Measurement of Staphylokinase by Enzyme-Linked Immunosorbent Assay Using Monoclonal Antibodies
}

\author{
Akito Mike, ${ }^{*}$ Yoko OHYa, Makoto Ohwaki, Masashi SAKaI, Tomoyuki SaKo, \\ Tsunekazu WatanaBe, and Teruo YoKoKURA \\ Yakult Central Institute for Microbiological Research, 1796 Yaho, Kunitachi-shi, Tokyo 186, Japan. \\ Received December 10, 1993; accepted January 26, 1994
}

\begin{abstract}
Hybridoma clones producing monoclonal antibodies specific for staphylokinase were isolated. A competitive assay revealed that the monoclonal antibodies studied could be divided into at least two groups. Representatives of these groups, AS22 and B3E6, recognized quite different epitopes on staphylokinase. This finding led us to develop an assay system for the quantitative analysis of staphylokinase by enzyme-linked immunosorbent assay using AS22 as the capturing antibody and biotinylated B3E6 as the "detector". The lower limit of sensitivity of the assay was $20 \mathrm{pg}$ of staphylokinase per $\mathrm{ml}$. The assay exhibited good reproducibility, with values of 5.8 and $3.8 \%$ for the intraand inter-assay coefficients of variation, respectively. Staphylokinase could be assayed in the presence of human plasma when the plasma was diluted more than 320 -fold, and the measurement was unaffected by the presence of physiological concentrations of human plasminogen. Hence, this assay was considered useful for the detection and quantification of staphylokinase in clinical samples.
\end{abstract}

Keywords staphylokinase; monoclonal antibody; ELISA

Staphylokinase (SAK) is a plasminogen activator produced by Staphylococcus aureus. ${ }^{1)}$ Thanks to the successful cloning of the sak gene and the production of recombinant $\mathrm{SAK},{ }^{2)}$ study of the thrombolytic activity of SAK has advanced greatly. Like streptokinase (SK), SAK is an indirect-type plasminogen activator which forms a complex with plasminogen that then activates other plasminogen molecules. ${ }^{3)}$ However, it leads to more preferential degradation of fibrin than SK in both in vitro and in vivo assay systems. ${ }^{4,5)}$ Recent reports have suggested that SAK might be a promising thrombolytic agent, ${ }^{6,7)}$ although, there is still much to be learned about its structure-activity behavior. ${ }^{8)}$

A monoclonal antibody ( $\mathrm{mAb}$ ) secreted by a hybridoma is a homogeneous antibody that recognizes an antigen at a specific antigenic determinant site (epitope). ${ }^{9)}$ It can be a valuable tool for the specific measurement of thrombolytic drugs and the elucidation of their structure-activity correlations. ${ }^{10,11)}$ We have produced mAbs that recognize SAK and examined the epitopes for these antibodies. As a result, at least two groups of $\mathrm{mAbs}$ have been identified. Using two antibodies which recognized distinct antigenic determinants on SAK, we have succeeded in developing a highly sensitive ELISA for measuring SAK.

\section{MATERIALS AND METHODS}

Materials Recombinant SAK was produced using an $E$. coli strain carrying the $s a k$ gene and purified according to the method of Sako. ${ }^{12)}$ Human plasma was purchased from Oriental Yeast (Tokyo, Japan) and human plasminogen was obtained from Kabi Vitrum AB (Stockholm, Sweden).

Cells A mouse myeloma cell line, P3-NS-1/1-Ag4-1 (NS-1), was provided by Dr.K.Yasui of the Tokyo Metropolitan Institute for Neuroscience (Tokyo, Japan). NS-1 cells and hybridoma cells were cultured in RPMI1640 medium (SIGMA, U.S.A.) containing $15 \%$ fetal calf serum (Nippon M.B.A., Japan).

Immunization Purified SAK $(100 \mu \mathrm{g})$ was administered intraperitoneally together with Freund's complete adjuvant to BALB/c mice (Japan SLC). Two weeks later, an additional immunization was performed in a similar manner. The anti-SAK antibody titer in the sera of the immunized mice was determined by ELISA (described later), and $25 \mu \mathrm{g}$ SAK was administered via the tail vein for the final immunization, $3 \mathrm{~d}$ before cell fusion.

Production of mAb Basically, the method of $\mathrm{Oi}$ and Herzenberg was used. ${ }^{13)}$ Splenocytes from the immune mice were mixed with NS-1 cells (splenocytes: NS-1 cells $=4: 1$ ), and then treated with polyethylene glycol (molecular weight, approx. 6000, dissolved in RPMI1640 to give a concentration of $45 \%(\mathrm{w} / \mathrm{v}))$. The mixture was seeded into 96-well culture plates, at $1 \times 10^{5}$ splenocytes/ well, and the plates incubated at $37^{\circ} \mathrm{C}$ under $7 \% \mathrm{CO}_{2}$ and $93 \%$ air. The anti-SAK antibody titer in the culture supernatant was determined by ELISA (described later), and the cells from the wells exhibiting the requierd antibody titer were cloned by limiting dilution.

Hybridoma cells, isolated and cloned in this way, were inoculated intraperitoneally into BALB/c mice (pretreated with pristane, 2,6,10,14-tetramethylpentadecane, Aldrich, U.S.A.), and then the ascitic fluid recovered from these mice was purified using a protein $\mathrm{G}$ column (MAbTrap G; Pharmacia LKB, Uppsala, Sweden). The eluates obtained (IgG fraction) were used as mAbs.

Biotinylation of $\mathbf{m A b} \mathrm{mAbs}$ were labeled with biotin by means of a biotinylation kit (Amersham, UK).

Measurement of the Anti-SAK Antibody (ELISA1) ELISA was carried out basically by the method of Voller et $a l^{14)}$ with a slight modification, as follows. Firstly, $2 \mu \mathrm{g} / \mathrm{ml} \mathrm{SAK}$ diluted with a sodium carbonate buffer $(\mathrm{pH}$ 9.6) was added to a 96-well polystyrene immunoplate (NUNC, Denmark; $100 \mu \mathrm{l} /$ well). The plate was allowed to stand at $4{ }^{\circ} \mathrm{C}$ overnight for adsorption of 
the antigen to take place, and then each well was washed with phosphate-buffered saline (PBS) containing $0.05 \%$ Triton X-100 (wash solution). A control plate with no adsorbed antigen was treated with $1 \%$ bovine serum albumin (BSA) (dissolved in sodium carbonate buffer) $\left(120 \mu \mathrm{l} /\right.$ well). The plate was then incubated at $37^{\circ} \mathrm{C}$ for $90 \mathrm{~min}$, washed, and mouse serum or $\mathrm{mAb}$ diluted appropriately with wash solution containing $1 \%$ BSA (diluting solution) was added $(90 \mu \mathrm{l} /$ well). The plate was again incubated at $37^{\circ} \mathrm{C}$ for $90 \mathrm{~min}$, then washed, and the peroxidase-labeled anti-mouse immunoglobulin antibody (Cappel, U.S.A.), diluted 1/1000 with diluting solution, was added $(100 \mu \mathrm{l} /$ well). Again, the reaction was allowed to proceed at $37^{\circ} \mathrm{C}$ for $90 \mathrm{~min}$, and then the plate was washed and the substrate solution added $(100 \mu \mathrm{l} /$ well $)$. The substrate solution was freshly prepared by dissolving $20 \mathrm{mg}$ $o$-phenylenediamine in $50 \mathrm{ml}$ of citrate buffer (pH 5.0), followed by the addition of hydrogen peroxide solution to give a final concentration of $0.006 \%(\mathrm{v} / \mathrm{v})$. The plate was allowed to stand at $37^{\circ} \mathrm{C}$ for $10 \mathrm{~min}$ and then the reaction was terminated by adding $2.5 \mathrm{M}$ sulfuric acid. Finally, the absorbance at $492 \mathrm{~nm}$ was determined using a Titertek Multiscan (Flow, Finland).

Analysis of mAbs Epitopes (ELISA-2) The competitive ELISA method was used to investigate the epitopes for the mAbs. In this assay, immunoplates to which SAK had been adsorbed were prepared as for ELISA-1. A mixture, containing one biotin-labeled $\mathrm{mAb}$ at a fixed titer and another unlabeled $\mathrm{mAb}$ at differnt titers, was added to the immunoplate $(90 \mu \mathrm{l} /$ well) and the reaction allowed to proceed at $37^{\circ} \mathrm{C}$ for $90 \mathrm{~min}$. After washing the plate, peroxidase-labeled streptavidin (Zymed, U.S.A.) diluted $1 / 2000$ with diluting solution was added to the plate $(100 \mu 1 /$ well), and the reaction was again allowed to proceed at $37^{\circ} \mathrm{C}$ for $60 \mathrm{~min}$. Then the plate was washed and the substrate solution added. The procedure after that was the same as for ELISA-1. The extent to which the binding of biotin-labeled $\mathrm{mAb}$ to SAK was blocked by unlabeled $\mathrm{mAb}$ was calculated as follows:

Inhibition (\%)

$$
=\left(1-\frac{\begin{array}{c}
\text { absorbance of the mixture of } \\
\text { biotinylated and unlabeled } \mathrm{mAbs}
\end{array}}{\text { absorbance of biotinylated } \mathrm{mAb} \text { alone }}\right) \times 100
$$

Measurement of SAK with mAbs (ELISA-3) SAK was measured by the ELISA sandwich method involving the use of two kinds of mAbs. The immunoplate was allowed to adsorb $10 \mu \mathrm{g} / \mathrm{ml}$ of one mAb, then SAK or SAK diluted with PBS containing human plasma or human plasminogen was added to the plate, followed by incubation at $37^{\circ} \mathrm{C}$ for $90 \mathrm{~min}$. After washing the plate, another $\mathrm{mAb}$ labeled with biotin was added and the reaction was again allowed to proceed at $37^{\circ} \mathrm{C}$ for $90 \mathrm{~min}$. After washing the plate, peroxidase-labeled streptavidin was added. The procedure after that was the same as for ELISA-2.

\section{RESULTS}

Splenocytes from SAK-immunized mice were fused with NS-1 mouse myeloma cells and the anti-SAK antibody in the culture was detected by ELISA. Hybridomas occurred in $322(55.9 \%)$ of the 576 wells and anti-SAK antibody was detected in 40 of these 322 wells. Cloning by limiting dilution yielded 11 anti-SAK antibody-producing hybridoma clones. The isotype of all the mAbs was IgG1.

Four of the above anti-SAK hybridoma clones and the B3E6 clone (previously reported by Sako and Tsuchida ${ }^{15)}$ ) were used for competitive ELISA in order to analyse the epitopes recognized by $\mathrm{mAbs}$. Each $\mathrm{mAb}$ was purified to an IgG fraction and a part of this fraction was labeled with biotin. Then, the inhibition of the binding of a biotinylated $\mathrm{mAb}$ to SAK by an unlabeled $\mathrm{mAb}$ was measured. With this assay, it was possible to determine whether mAbs bound to the same region of SAK. In all reactions, the binding of the biotin-labeled antibody was strongly blocked by the unlabeled antibody itself and the inhibition of this binding depended on the concentration of the unlabeled antibody (Fig. $1 \mathrm{~A}-\mathrm{E}$ ). AS102 was as strongly inhibited by AS504 as it was by itself, and vice versa; both AS102 and AS504 were also strongly inhibited by AS22, and moderately inhibited by AS17 and B3E6 (Fig. 1C and D). These results indicate that both the recognition sites on SAK, and the binding properties of AS102 and AS504 are the same or very similar. AS22 strongly blocked the binding of AS102 and AS504, and its binding was moderately blocked by AS102 and AS504. In addition, the binding of AS102 or AS504 to SAK was strongly inhibited by AS22, while the binding of AS22 to SAK was only moderately inhibited by AS102 or AS504 (Fig. 1B, C and D). It seems likely that the recognition site of AS22 is close to that of AS102 and AS504, and that the binding of AS22 to SAK is stronger than that of AS102 and AS504. The binding of AS17 to SAK was strongly inhibited by B3E6, but not vice versa (Fig. 1A and E) while AS22 and B3E6 did not block each other's binding to SAK (Fig. 1B and E). These competitive results are summarized in Fig. 2.

Since AS22 and B3E6 do not prevent each other from binding to SAK, their epitopes on SAK must be quite different. Thus, we attempted to assay SAK quantitatively with these two mAbs. When AS22 was used as the antigen to capture the antibody responsible for the adsorption of another antigen to the immunoplate, and B3E6 as the biotin-labeled antibody acting as the "detector" (Fig. 3), SAK was detected with great sensitivity: the limit of detection of SAK in this assay system was approximately $20 \mathrm{pg} / \mathrm{ml}$. Similar results were obtained when B3E6 was used as the "captor" and AS22 as the. "detector". When the same antibody was used as both "captor" and "detector", SAK could not be detected.

The intra-assay coefficient of variation of the assay was $5.8 \%$ ( 5 dilutions, 5 measurements at each dilution) and the inter-assay (day-to-day) coefficient of variation was $3.8 \%$ (5 dilutions, 5 measurements). This confirms the high reproducibility of the method.

SAK diluted with human plasma $(1: 5,1: 20,1: 80$ and $1: 320)$ and with diluting buffer alone was assayed by the sandwich ELISA method described above. The quantitative detection of SAK in solutions containing human plasma was affected severely when the concentration of plasma was high (Fig. 4). This inhibitory effect of 

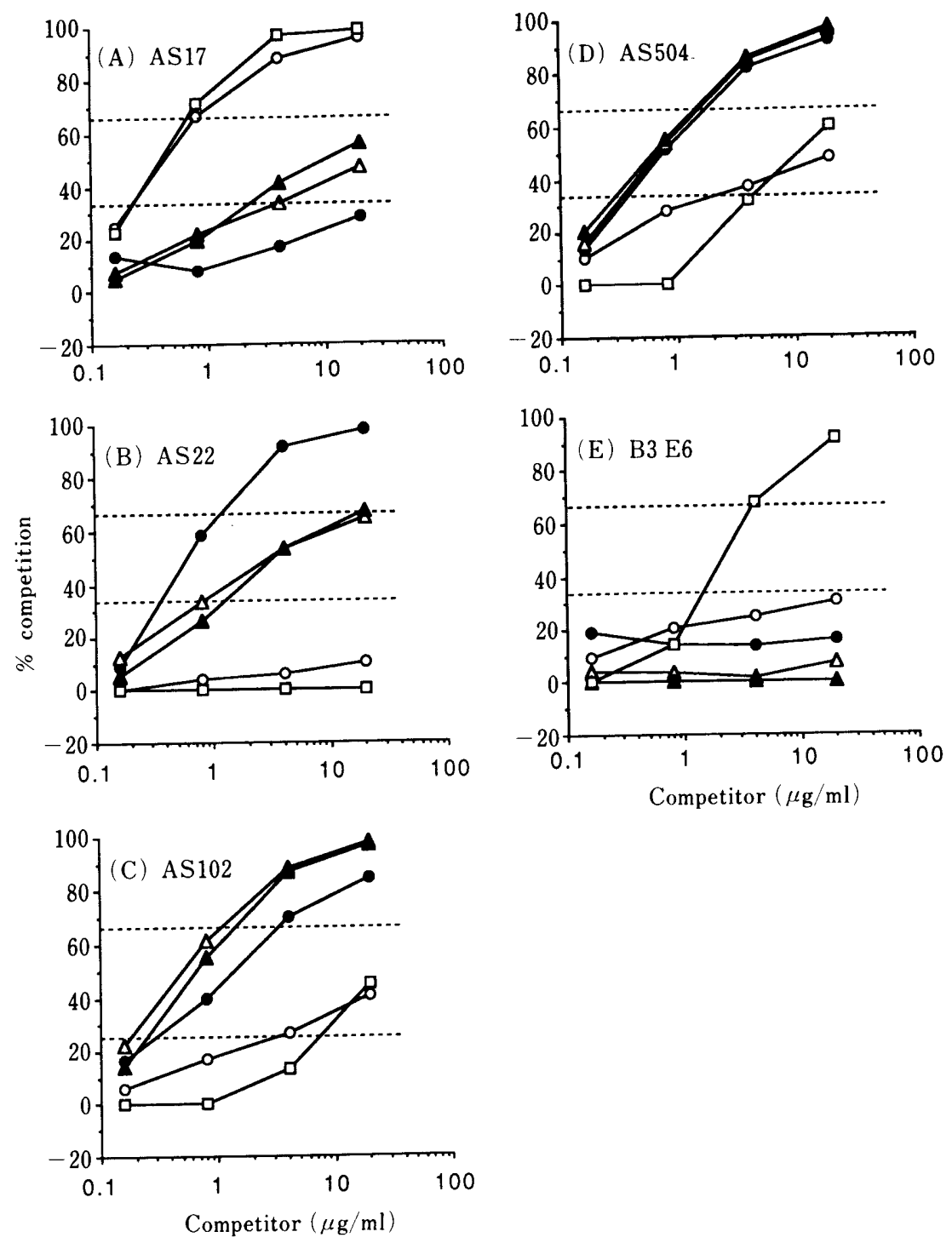

Fig. 1. Competitive ELISA with Unlabeled Purified mAbs against Biotinylated mAbs

Unlabeled competitor mAbs were serially diluted 5-fold, from 20 to $0.16 \mu \mathrm{g} / \mathrm{ml}$, and saturating quantities of biotinylated mAbs were added. Plates were coated with

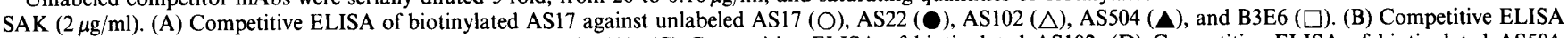
of biotinylated AS22. Symbols for unlabeled mAbs, are as in (A). (C) Competitive ELISA of biotinylated AS102. (D) Competitive ELISA of biotinylated AS504. (E) Competitive ELISA of biotinylated B3E6.

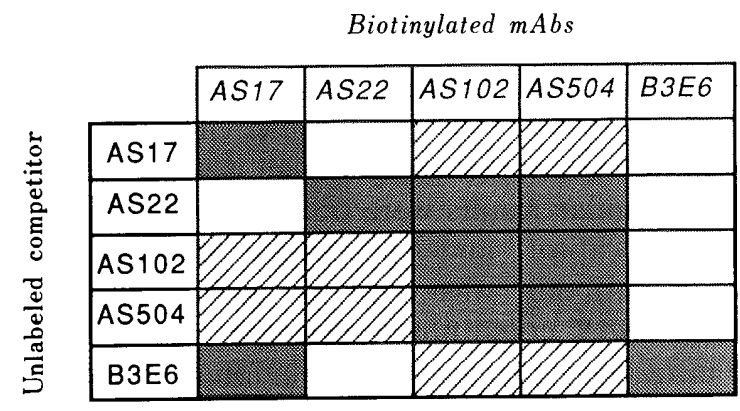

relative strength of competition

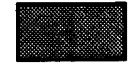

strong $>67 \%$

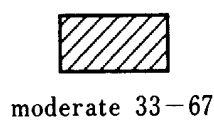

moderate $33-67 \%$

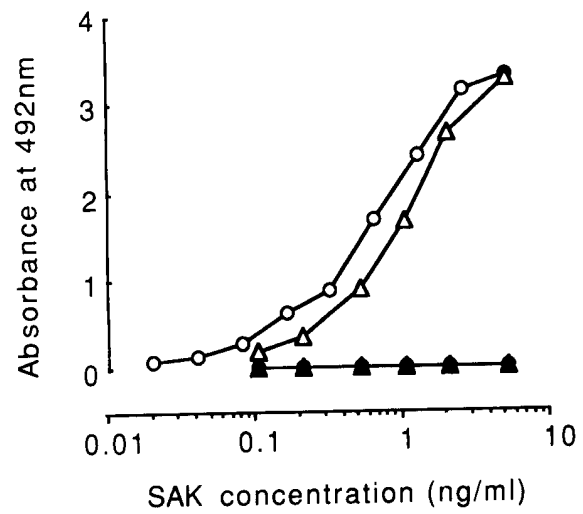

Fig. 3. Dose-Response Curves for SAK on Sandwich ELISA Involving Binding of Two MAbs to SAK, Dissolved in PBS (Final Concentrations, 0.02 to $5 \mathrm{ng} / \mathrm{ml}$ )

captor $\mathrm{mAb}$ detector $\mathrm{mAb}$

$$
\begin{aligned}
& \text { AS22 - }- \text { B3E6 } \\
& \text { AS22 - - AS22 } \\
& \text { B3E6 - }- \text { AS22 } \\
& \text { B3E6- }- \text { B3E6. }
\end{aligned}
$$




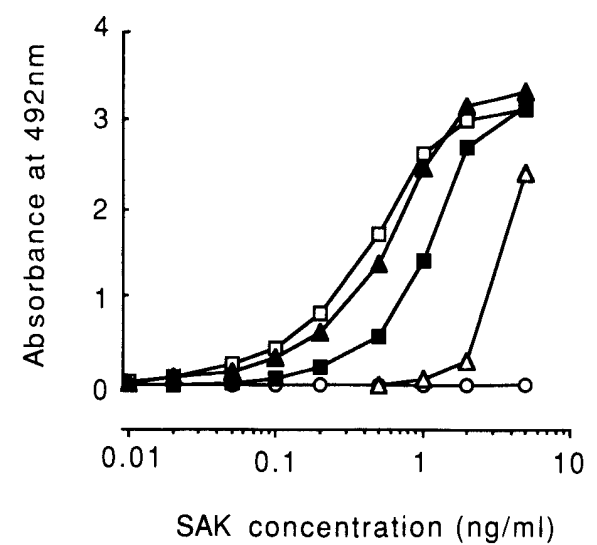

Fig. 4. Influence of Human Plasma on the Detection of SAK

SAK levels (range, 0.01 to $5 \mathrm{ng} / \mathrm{ml}$ ) were measured after dilution of plasma $(1: 5$, $0 ; 1: 20, \triangle ; 1: 80, \square ; 1: 320, \mathbf{\Delta})$, and in PBS $(\square)$.

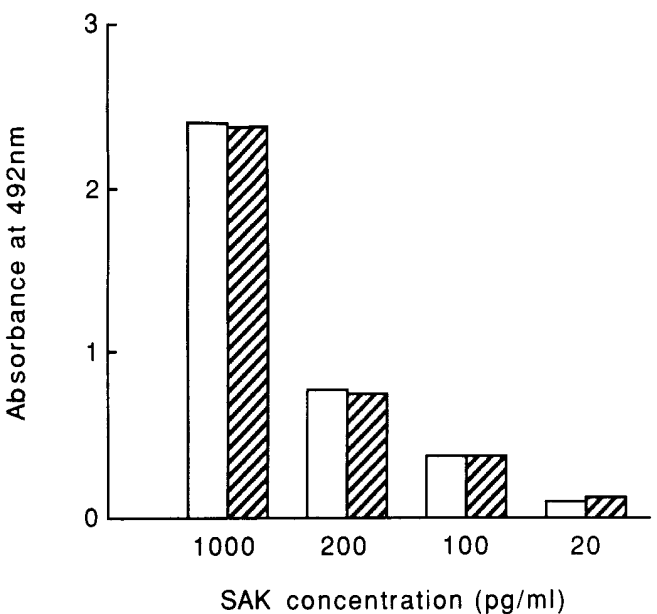

Fig. 5. Interference Due to Human Plasminogen in the Detection of SAK

SAK levels (range, 20 to $1000 \mathrm{pg} / \mathrm{ml}$ ) were measured in the presence (final concentration, $1.4 \mu \mathrm{M})$ or absence of plasminogen.

, with plasminogen $(1.4 \mu \mathrm{M}) ; \square$, without plasminogen

human plasma was completely eliminated when the plasma was diluted 320 -fold.

However, measurement of SAK with this assay system was unaffected by the presence of physiological concentrations $(1.4 \mu \mathrm{M})$ of human plasminogen (Fig. 5), showing that the inhibition of antibody binding by human plasma was not due to the presence of plasminogen.

\section{DISCUSSION}

In the present study, we isolated mouse hybridoma clones producing mAbs against SAK and developed a SAK assay system involving two mAbs.

The relationship between the epitopes for the mAbs was investigated by means of a competitive assay. AS102 and AS504 showed quite similar characteristics in this assay, indicating that the epitopes for these two antibodies are the same or very similar one another. The similar inhibitory effects of AS22 on the binding of AS102 and AS504 to themselves suggests that the epitope for AS22 is very close to that of AS102 and AS504. However, AS102 and AS504 only weakly inhibited the binding of AS22. This probably reflects the difference in the affinity for SAK exhibited by
AS22 and the other antibodies. The relationship between AS17 and B3E6 is similar. The epitopes for these two $\mathrm{mAbs}$ seem to be close to each other, whereas B3E6 binds to SAK more strongly than AS17. Hence, the mAbs analyzed in this study can be divided into two groups: one includes AS17 and B3E6 and the other AS22, AS102 and AS504. As shown in Figs. 1 and 3, AS22 and B3E6 recognize quite different epitopes on SAK.

An antibody immunoassay can detect the target material specifically, simply and rapidly. ${ }^{16)}$ In the present study, we produced mAbs against SAK and established a SAK assay based on sandwich ELISA, involving two clones with different epitopes, AS22 and B3E6. This assay system is not only highly sensitive for SAK, but is also able to detect SAK in human plasma. However, since the sensitivity for SAK was lowered in human plasma, we attempted to detect SAK in the presence of physiological concentrations of human plasminogen alone. It was clear that the SAK assay was unaffected by the addition of plasminogen and so the epitopes for the mAbs on SAK are thought not to be plasminogen-binding sites. Information about other factors in human plasma that interfere with the binding of $\mathrm{mAb}$ to SAK is not available.

Many publications have described the quantitation of thrombolytic agents, particularly tissue-type plasminogen activator (t-PA), with mAbs. Various mAbs against t-PA or SK are now used for elucidating their mechanisms of action and pharmacokinetics, or to investigate their antigenicity. ${ }^{10,11,17)}$ We have produced mAbs against SAK and succeeded in developing a SAK assay using these antibodies. In addition, multiple mAbs recognizing different sites on the SAK molecule, were obtained. These mAbs will be a useful tool for elucidating the structure-activity relationships exhibited by SAK.

Acknowledgment We thank Dr. T.Osawa for helpful discussions.

\section{REFERENCES}

1) R. R. Maddison, Proc. Sci. Exp. Med., 33, 209 (1935).

2) T. Sako, S. Sawaki, T. Sakurai, S. Ito, Y. Yoshizawa, I. Kondo, Mol. Gen. Genet., 252, 271 (1983).

3) B. Kowalska-Loth, K. Zakrzewski, Acta Biochim., 22, 327 (1975).

4) O. Matsuo, K. Okada, H. Fukao, Y. Tomioka, S. Ueshima, M. Watanuki, M. Sakai, Blood, 76, 925 (1990).

5) M. Sakai, M. Watanuki, O. Matsuo, Biochem. Biophys. Res. Commun., 162, 830 (1989).

6) D. Collen, F. D. Cock, J. H. Stassen, Circulation, 87, 996 (1993).

7) D. Collen, F. V. Welf, Circulation, 87, 1850 (1993).

8) M. Sakai, Nippon Nogeikagaku Kaishi, 66, 1227 (1992).

9) G. Kohler, C. Milstein, Nature (London), 256, 495 (1975).

10) P. Holvoet, H. Cleemput, D. Collen, Thromb. Haemostas., 54, 684 (1985).

11) T. Kaizu, K. Kojima, K. Iwasaki, T. Yamashita, Thromb. Res., 40, 91 (1985).

12) T. Sako, Eur. J. Biochem., 149, 557 (1985).

13) V. T. Oi, L. A. Herzenberg, "Selected Methods in Cellular Immunology," W. H. Freeman \& Co., San Francisco, 1979, pp. $351-371$.

14) A. Voller, D. E. Bidwell, A. Bartlett, Bull. WHO, 53, 55 (1976).

15) T. Sako, N .Tsuchida, Nucleic Acids Res., 11, 7679 (1983).

16) O. Matsuo, K. Kato, C. Matsuo, T. Matsuo, Anal. Biochem., 135, 58 (1983).

17) G. L. Reed, P. Kussie, B. Parhami-Seren, J. Immunol., 150, 4407 (1993). 